\title{
Fenologia da Trapoeraba como Indicador para Tolerância ao HERBICIDA GLYPHOSATE ${ }^{1}$
}

\author{
Phenology of Bengal Dayflower as Indicator of Glyphosate Tolerance \\ DIAS, A.C.R. ${ }^{2}$, CARVALHO, S.J.P. ${ }^{3}$ e CHRISTOFFOLETI, P.J. ${ }^{4}$
}

\begin{abstract}
RESUMO - Rotineiramente, tem sido desconsiderada a contribuição do estádio de desenvolvimento das espécies de plantas daninhas nas análises de tolerância ou resistência a herbicidas, o que pode resultar em divergências entre a pesquisa teórica e a aplicação prática dos dados. Nesse sentido, este trabalho foi desenvolvido com o objetivo de avaliar a resposta biológica da trapoeraba (Commelina benghalensis), comparativamente ao capimmarmelada (Brachiaria plantaginea), a aplicações de doses do herbicida glyphosate, em seis estádios fenológicos. Dois experimentos foram desenvolvidos em casa de vegetação, submetendo-se plantas de trapoeraba e capim-marmelada ao esquema fatorial de tratamentos $9 \times 6$, em que nove foram as doses de glyphosate e seis foram os estádios fenológicos das plantas daninhas, variáveis entre a emissão da primeira folha definitiva e o início do florescimento. Por meio do emprego de curvas de dose-resposta e de regressões polinomiais, concluiu-se que o estádio de desenvolvimento da trapoeraba contribui significativamente para o grau de tolerância da espécie ao herbicida glyphosate, de modo que, comparativamente ao capim-marmelada, plantas de trapoeraba tornam-se quatro vezes mais tolerantes ao glyphosate a cada dez unidades de desenvolvimento fenológico na escala BBCH. Essas considerações possuem importante aplicabilidade prática, justificando medidas de controle químico em estádios iniciais do crescimento da trapoeraba.
\end{abstract}

Palavras-chave: Commelina benghalensis, Brachiaria plantaginea, fenologia, crescimento, glifosato, modelagem.

\begin{abstract}
The importance of the phenological development of weeds has been frequently ignored when analyzing plant-herbicide tolerance or resistance, what may result on divergences between theoretical research and practical data application. Thus, this work was carried out to evaluate the biological response of Bengal dayflower (Commelina benghalensis), comparatively to alexandergrass (Brachiaria plantaginea), to the application of different rates of glyphosate, at six phenological stages. Two experiments were developed under greenhouse conditions, with Bengal dayflower and alexandergrass plants being submitted to a $9 \times 6$ factorial scheme, where nine treatments were the glyphosate rates and six, the weed phenological stages, varying between unfolding of the first true leaf and the beginning of flowering. Using dose-response curves and polynomial regressions, it could be concluded that the Bengal dayflower phenological stages significantly contribute to the species' degree of glyphosate-tolerance, since, compared to alexandergrass, Bengal dayflower plants became fourtimes more tolerant to glyphosate at every ten units of phenological development at the $B B C H$ scale. These considerations have an important practical applicability, justifying chemical control measurements at the initial growth stages of Bengal dayflower.
\end{abstract}

Keywords: Commelina benghalensis, Brachiaria plantaginea, phenology, growth, glyphosate, modeling.

1 Recebido para publicação em 9.5.2012 e aprovado em 14.10.2012 .

Parte da Dissertação da primeira autora, apresentada à Esalq/USP, para obtenção do título de Mestre em Agronomia/Fitotecnia.

2 Engâ-Agr ${ }^{\mathrm{a}}$., Mestre e Doutora em Fitotecnia pela Escola Superior de Agricultura "Luiz de Queiroz" - Esalq/USP, <anacarolina.r.dias@gmail.com>; ${ }^{3}$ Professor, Doutor, Instituto Federal do Sul de Minas, Campus Machado, Caixa Postal 1004, 37750-971 Machado-MG, <sjpcarvalho@yahoo.com.br>; ${ }^{4}$ Professor Associado, Esalq/USP, <pjchrist@esalq.usp.br>.

Planta Daninha, Viçosa-MG, v. 31, n. 1, p. 185-191, 2013 


\section{INTRODUÇÃO}

O glyphosate é um herbicida derivado do aminoácido glicina e tem como mecanismo de ação a inibição da 5-enol-piruvil-shikimato3-fosfato sintase (EPSPs), enzima responsável por uma das etapas de síntese dos aminoácidos aromáticos triptofano, fenilalanina e tirosina (Kruse et al., 2000; Zonetti et al., 2011). Não é seletivo, exceto para culturas geneticamente modificadas para resistência; possui ação sistêmica; não tem atividade residual no solo; e pode ser utilizado no controle de plantas daninhas anuais e perenes, bem como para dessecação, principalmente nas áreas de plantio direto e no manejo de plantas daninhas na linha de culturas perenes (Rodrigues \& Almeida, 2011).

Em áreas onde o herbicida glyphosate é utilizado com frequência, populações de trapoeraba (Commelina benghalensis) têm sido selecionadas, o que está associado à tolerância dessa espécie às aplicações desse herbicida (Rocha et al., 2007; Webster \& Grey, 2008; Dias et al., 2009; Maciel et al., 2011). Já o capimmarmelada (Brachiaria plantaginea) é uma planta daninha muito frequente em culturas anuais e perenes no Brasil, sendo muito competitiva quando presente em culturas anuais (Brighenti \& Oliveira, 2011), como soja e milho, provocando prejuízos consideráveis ao rendimento e à qualidade da produção (Dias et al., 2010; Pereira et al., 2010).

Nesse sentido, considera-se que o controle eficiente de plantas daninhas com o uso de herbicidas em pós-emergência depende, sobretudo, do estádio de desenvolvimento das plantas-alvo (Askew et al., 2000; Johnson \& Hoverstad, 2002; Fleck et al., 2008). O emprego inadequado do glyphosate para controlar a trapoeraba, em seus diferentes estádios fenológicos, pode promover gasto desnecessário de capital e energia química (dose demasiadamente elevada nos estádios iniciais) ou mesmo resultar em baixo controle (estádios mais avançados).

De acordo com Pereira et al. (2010), a utilização de doses elevadas de herbicidas vem sendo intensamente questionada nos últimos anos, em razão dos possíveis danos causados ao agroecossistema, do custo elevado e do aumento da pressão de seleção sobre biótipos resistentes a herbicidas. Entretanto, as doses recomendadas de alguns herbicidas podem ser reduzidas sem prejuízo para o controle das plantas daninhas e sem afetar o rendimento da cultura, o que possibilita manter o controle de plantas daninhas em níveis satisfatórios, sendoecologicamente mais corretas e economicamente viáveis.

Assim, para que o glyphosate seja usado de forma racional, visando aplicação de doses ajustadas aos diferentes estádios fenológicos dessa planta daninha, algumas ferramentas de manejo podem ser utilizadas, como os modelos matemáticos. Entre os modelos matemáticos existentes, diversos autores têm utilizado e recomendado as análises por curvas de dose-resposta para determinar a suscetibilidade ou resistência de plantas daninhas aos herbicidas (Streibig et al., 1993; Friensen et al., 1993; Madsen \& Jensen, 1995; Hall et al., 1998).

Em geral, considera-se que o modelo loglogístico possui vantagens em relação a outros métodos de análise, como, por exemplo, parâmetros de simples interpretação biológica. Contudo, a principal vantagem está relacionada com um dos termos integrantes da equação não linear, que é a estimativa do $\mathrm{GR}_{50}$, facilitando assim a comparação do nivel de resistência dos biótipos ou tolerância entre espécies testadas. $\mathrm{O} \mathrm{GR}_{50}$ é a dose do herbicida $\left(\mathrm{g} \mathrm{ha}^{-1}\right)$ necessária para proporcionar $50 \%$ de redução do crescimento da planta daninha (Christoffoleti, 2002; Christoffoleti \& LópezOvejero, 2008).

Por outro lado, o modelo logístico não contempla a influência do estádio de desenvolvimento da planta daninha no controle a ser obtido, o que, potencialmente, pode ser corrigido por análises que correlacionem dose, estádio fenológico e controle. Assim, este trabalho foi desenvolvido com o objetivo de avaliar a resposta biológica da trapoeraba (Commelina benghalensis), comparativamente ao capimmarmelada (Brachiaria plantaginea), a aplicações de doses do herbicida glyphosate em seis estádios fenológicos.

\section{MATERIAL E MÉTODOS}

Dois experimentos foram desenvolvidos em casa de vegetação de janeiro a julho de 
2008. As espécies estudadas (experimentos) foram: trapoeraba (Commelina benghalensis) e capim-marmelada (Brachiaria plantaginea). A segunda espécie foi estudada devido à sua elevada suscetibilidade ao glyphosate (Lorenzi, 2000; Roman et al., 2004), com o intuito de auxiliar na quantificação da resposta biológica da trapoeraba.

As sementes das plantas daninhas foram adquiridas na Cosmos Agrícola Produção e Serviços Ltda. Elas foram acondicionadas em saco de papel, em local seco, à temperatura ambiente, até a instalação do experimento. Inicialmente, as sementes foram colocadas para germinar em caixas plásticas com capacidade para $2 \mathrm{~L}$, preenchidas com substrato comercial Plantmax ${ }^{\circledR}$ (turfa + vermiculita + casca de pínus), e acondicionadas em câmara de germinação tipo $\mathrm{BOD}$, regulada à temperatura constante de $25^{\circ} \mathrm{C}$ e fotoperiodo de 14 horas de luz.

Após germinação e emergência, quatro plântulas de trapoeraba ou capim-marmelada foram transplantadas para cada parcela, em estádio fenológico de emissão da primeira folha verdadeira, ou seja, 10 na escala $\mathrm{BBCH}$ (Hess et al., 1997). As parcelas experimentais constaram de vasos plásticos com capacidade para 1,5 L, também preenchidos com substrato comercial (Plantmax ${ }^{\circledR}$ ), devidamente fertilizados com $100 \mathrm{mg}$ de $\mathrm{N}, \mathrm{P}_{2} \mathrm{O}_{5}$ e $\mathrm{K}_{2} \mathrm{O}$.

O delineamento experimental adotado foi o inteiramente ao acaso com quatro repetições. Para cada experimento (espécie), os tratamentos resultaram de esquema fatorial $9 \times 6$, em que nove foram as doses de glyphosate e seis foram os estádios fenológicos das plantas daninhas, ajustados à escala $\mathrm{BBCH}$. Para a trapoeraba, os estádios fenológicos registrados no momento das aplicações foram: primeira folha verdadeira - $\mathrm{BBCH} 10$; duas folhas verdadeiras $-\mathrm{BBCH} 12$; quatro folhas $-\mathrm{BBCH}$ 14; seis folhas e cinco ramos laterais $-\mathrm{BBCH}$ 25 ; oito folhas e sete ramos laterais $-\mathrm{BBCH}$ 34; e dez folhas e doze ramos laterais (início do florescimento) - BBCH 51. Para o capimmarmelada, os estádios fenológicos foram: primeira folha verdadeira-BBCH 10; três folhas verdadeiras - $\mathrm{BBCH} 13$; três folhas e três perfilhos - BBCH 23; três folhas e seis perfilhos $\mathrm{BBCH} 26$; cinco folhas e nove perfilhos $-\mathrm{BBCH}$ 33; e início de florescimento, com vinte perfilhos - BBCH 51. Os estádios fenológicos foram definidos quando $50 \%+1$ das plantas apresentaram determinada característica de desenvolvimento (Carvalho et al., 2005a). No momento das aplicações, quatro plantas de cada espécie foram amostradas e avaliadas também quanto à massa seca e área foliar.

Sendo D a dose recomendada do glyphosate ( $360 \mathrm{~g} \mathrm{ha}^{-1}$ ) para o capim-marmelada, os niveis do fator herbicida (doses), para ambos os experimentos, foram: 8D, 4D, 2D, D, 1/2D, 1/ 4D, $1 / 8 \mathrm{D}, 1 / 16 \mathrm{D}$ e ausência do herbicida, o que equivale a $2.880,1.440,720,360,180,90$, 45, 22,5 e $0 \mathrm{~g} \mathrm{ha}^{-1}$. Todas as aplicações de glyphosate foram realizadas em câmara de pulverização, utilizando uma ponta do tipo leque, com jato plano, modelo XR 80.02, calibrada para volume de calda correspondente a $200 \mathrm{~L} \mathrm{ha}^{-1}$. Depois da aplicação, os vasos foram acondicionados em casa de vegetação, sem irrigação por 24 horas, para garantir a absorção do herbicida; após esse período, todos os vasos receberam uma lâmina d'água proporcional a uma precipitação de $10 \mathrm{~mm}$, sendo irrigados diariamente.

A fim de reduzir o efeito de avaliações subjetivas, avaliou-se somente a massa seca residual aos 28 dias após a aplicação (DAA). A massa seca foi obtida a partir da colheita de todo o material vegetal remanescente da parte aérea das plantas nas parcelas, com posterior secagem em estufa a $70{ }^{\circ} \mathrm{C}$ por 96 horas. Inicialmente, para cada experimento, os dados de massa seca foram submetidos à análise da variância com aplicação do teste $\mathrm{F}$. Em cada estádio fenológico, as doses de glyphosate foram ajustadas a modelos de regressão não lineares do tipo log-logístico (Seefeldt et al., 1995):

$$
y=P \min +\frac{a}{\left[1+\left(\frac{x}{b}\right)^{c}\right]}
$$

em que: $y=$ massa seca residual; $x=$ dose do herbicida; e Pmín, $a, b$ e $c=$ parâmetros da curva, de modo que Pmín é o limite inferior da curva (ponto mínimo), $a$ é a diferença entre o ponto máximo e mínimo da curva, $b$ é a dose que proporciona $50 \%$ de resposta da variável e $c$ é a declividade da curva. 
De forma a complementar as análises, calcularam-se matematicamente os valores de $\mathrm{GR}_{50}$, utilizando-se o princípio da equação inversa, conforme discutido por Carvalho et al. (2005b). Posteriormente, os sucessivos valores de $\mathrm{GR}_{50}$ foram ajustados a modelos polinomiais de terceiro grau, correlacionando as variáveis de desenvolvimento vegetal com o aumento de sua tolerância a herbicidas. Por último, procedeu-se à análise conjunta dos dados, calculando-se o Fator de Tolerância da trapoeraba $(F t)$ :

$$
F t=\frac{G R_{50} \text { Trapoeraba }}{G R_{50} \text { Capim-marmelada }}
$$

$\mathrm{O}$ ajuste do fator de tolerância foi obtido por meio de regressão de primeiro grau, após divisão dos sucessivos valores de $\mathrm{GR}_{50}$ da trapoeraba pelos de $\mathrm{GR}_{50}$ do capim-marmelada, de modo a se compreender a evolução da tolerância da primeira ao glyphosate.

\section{RESULTADOS E DISCUSSÃO}

Para ambas as espécies, registrou-se o início de florescimento como estádio máximo para aplicação do herbicida, com ocorrência aos 51 e 63 DAS para trapoeraba e capimmarmelada, respectivamente (Tabela 1). Os ajustes da evolução de $\mathrm{GR}_{50}$ e do fator de tolerância foram feitos sempre com base na escala $\mathrm{BBCH}$ (eixo $x$ ). Essa opção foi consequência da facilidade de interpretação de dados fenológicos, aplicabilidade em condição de campo e, principalmente, da igualdade de amplitude dos eixos de desenvolvimento, com variação entre BBCH 10 e 51 (Tabela 1).

Quanto à massa seca residual, em ambos os experimentos, a aplicação do teste $F$ na análise da variância indicou a significância da interação dose-estádio fenológico $(\alpha=0,01)$, o que justificou a decomposição da interação com a análise de curvas de dose-resposta em cada estádio separadamente. Os parâmetros do modelo logístico para os dois experimentos, em todos os estádios, estão apresentados na Tabela 2. Houve elevado ajuste do modelo aos dados avaliados, exceto para a massa seca da trapoeraba em estádio BBCH 51 (início do florescimento). Por essa ocasião, não foram obtidos 50\% de redução de massa seca comparada à massa seca máxima, inviabilizando o uso do modelo. Nesse caso, mesmo a dose de $2.880 \mathrm{~g} \mathrm{ha}^{-1}$ de equivalente ácido de glyphosate não alcançou $50 \%$ de redução de massa, de modo que se considerou o $\mathrm{GR}_{50}$ deste estádio como maior que 2.880 (Tabela 2). Rocha et al. (2007) também registraram controle visual da trapoeraba abaixo de 50\% (23,7\%) aos 28 DAA,

Tabela 1 - Caracterização das plantas de trapoeraba e capim-marmelada no momento da aplicação das doses do herbicida glyphosate, em seis estádios fenológicos. Piracicaba-SP, 2008

\begin{tabular}{|c|c|c|c|c|}
\hline Estádio fenológico (EF) & $\begin{array}{c}\text { Escala } \\
\mathrm{BBCH}^{\underline{1}}\end{array}$ & $\begin{array}{c}\text { Dias após } \\
\text { semeadura }\end{array}$ & $\begin{array}{l}\text { Massa Seca } \\
\text { (g por planta) }\end{array}$ & $\begin{array}{c}\text { Área foliar } \\
\left(\mathrm{cm}^{2} \text { por planta }\right)\end{array}$ \\
\hline \multicolumn{5}{|c|}{ Trapoeraba - Commelina benghalensis } \\
\hline Uma folha & 10 & 14 & 0,01 & 0,41 \\
\hline Duas folhas & 12 & 17 & 0,04 & 0,55 \\
\hline Quatro folhas & 14 & 23 & 0,15 & 14,07 \\
\hline Seis folhas + cinco ramos & 25 & 30 & 0,28 & 91,18 \\
\hline Oito folhas + sete ramos & 34 & 38 & 3,26 & 305,01 \\
\hline Dez folhas + doze ramos (florescimento) & 51 & 51 & 5,03 & 538,89 \\
\hline \multicolumn{5}{|c|}{ Capim-marmelada - Brachiaria plantaginea } \\
\hline Uma folha & 10 & 9 & 0,01 & 0,32 \\
\hline Três folhas & 13 & 14 & 0,12 & 1,41 \\
\hline Três folhas + três perfilhos & 23 & 22 & 1,50 & 24,98 \\
\hline Três folhas + seis perfilhos & 26 & 27 & 2,16 & 33,84 \\
\hline Cinco folhas + nove perfilhos & 33 & 41 & 7,32 & 107,08 \\
\hline Seis folhas + vinte perfilhos & 51 & 63 & 14,25 & 862,63 \\
\hline
\end{tabular}

1/ Hess et al. (1997). 
Tabela 2 - Parâmetros do modelo logístico, coeficiente de determinação $\left(\mathrm{R}^{2}\right)$, teste $\mathrm{F}$ e $\mathrm{GR}_{50}$ para massa seca de trapoeraba e capimmarmelada submetidas à aplicação de doses do herbicida glyphosate, em seis estádios fenológicos (BBCH). Piracicaba-SP, 2008

\begin{tabular}{|c|c|c|c|c|c|c|c|}
\hline \multirow{2}{*}{$\mathrm{BBCH}^{1 /}$} & \multicolumn{4}{|c|}{ Parâmetros - Modelo Logístico ${ }^{\underline{2} /}$} & \multirow{2}{*}{$\mathrm{R}^{2}$} & \multirow{2}{*}{$\mathrm{F}$} & \multirow{2}{*}{$\mathrm{GR}_{50}$} \\
\hline & Pmín & $\mathrm{a}$ & $\mathrm{b}$ & $\mathrm{c}$ & & & \\
\hline \multicolumn{8}{|c|}{ Trapoeraba - Commelina benghalensis } \\
\hline 10 & 0,023 & 0,300 & 14,663 & 2,237 & 0,989 & $175,430 *$ & 15,847 \\
\hline 12 & 0,287 & 3,174 & 423,045 & 8,228 & 0,986 & $116,655^{*}$ & 420,718 \\
\hline 14 & $-0,561$ & 8,906 & 196,501 & 1,064 & 0,945 & $28,916^{*}$ & 195,839 \\
\hline 25 & 2,736 & 17,363 & 826,464 & 3,056 & 0,974 & $61,981^{*}$ & 848,412 \\
\hline 34 & 8,208 & 25,472 & 146,062 & 0,742 & 0,977 & $72,321 *$ & 380,601 \\
\hline 51 & \multicolumn{6}{|c|}{ sem ajuste } & $>2880,000$ \\
\hline \multicolumn{8}{|c|}{ Capim-marmelada - Brachiaria plantaginea } \\
\hline 10 & $-0,049$ & 2,225 & 28,214 & 1,074 & 0,994 & $300,222 *$ & 26,990 \\
\hline 13 & $-0,008$ & 6,032 & 41,511 & 1,629 & 0,990 & $159,445^{*}$ & 40,807 \\
\hline 23 & $-0,429$ & 16,569 & 53,703 & 1,358 & 0,973 & $60,078^{*}$ & 49,619 \\
\hline 26 & 12,170 & 19,207 & 25,422 & 1,534 & 0,998 & $975,103^{*}$ & 67,495 \\
\hline 33 & 12,859 & 53,995 & 35,830 & 1,693 & 0,998 & $1319,839 *$ & 47,682 \\
\hline 51 & 28,933 & 52,314 & 44,348 & 1,108 & 0,994 & $263,638^{*}$ & 135,187 \\
\hline
\end{tabular}

${ }_{1}^{1 /}$ Escala Fenológica - BBCH (Hess et al., 1997); ${ }^{2 /}$ Modelo: $\mathrm{y}=$ Pmín $+\left(\mathrm{a} /\left(1+(\mathrm{x} / \mathrm{b})^{\mathrm{c}}\right)\right)$; * Teste $\mathrm{F}$ significativo a $1 \%$.

após aplicação de $960 \mathrm{~g} \mathrm{ha}^{-1}$ de glyphosate. Lacerda \& Victória Filho (2004) também constataram a inviabilidade de determinar a dose necessária para controle de C. benghalensis, como observado na Tabela 2, uma vez que as doses aplicadas de glyphosate $(0,720$, $960,1.200,1.440,1.680$ e $1.920 \mathrm{~g} \mathrm{ha}^{-1}$ ) não foram suficientes para atingir niveis de $50 \%$ de controle. O fato de esta planta apresentar baixo controle com aplicações de doses altas foi explicado por Monquero et al. (2004), que encontraram o metabólito ácido aminometilfosfônico (AMPA) em plantas de trapoeraba, evidenciando a capacidade da espécie em converter a molécula de glyphosate em compostos menos tóxicos, provando que a absorção diferencial e o metabolismo do glyphosate são os mecanismos envolvidos na maior tolerância da espécie ao herbicida.

Para ambas as espécies, à medida que se observou o desenvolvimento das plantas, houve redução dos níveis de controle, ou seja, registrou-se necessidade de maior dose de herbicida para obtenção de $\mathrm{GR}_{50}$ (Figura 1). Ainda, as doses necessárias para obtenção de $\mathrm{GR}_{50}$ da trapoeraba foram superiores àquelas necessárias para controle do capim-marmelada (Tabela 2). No presente trabalho, mesmo quando em início de florescimento, o $\mathrm{GR}_{50}$ do capim-marmelada foi obtido com doses de
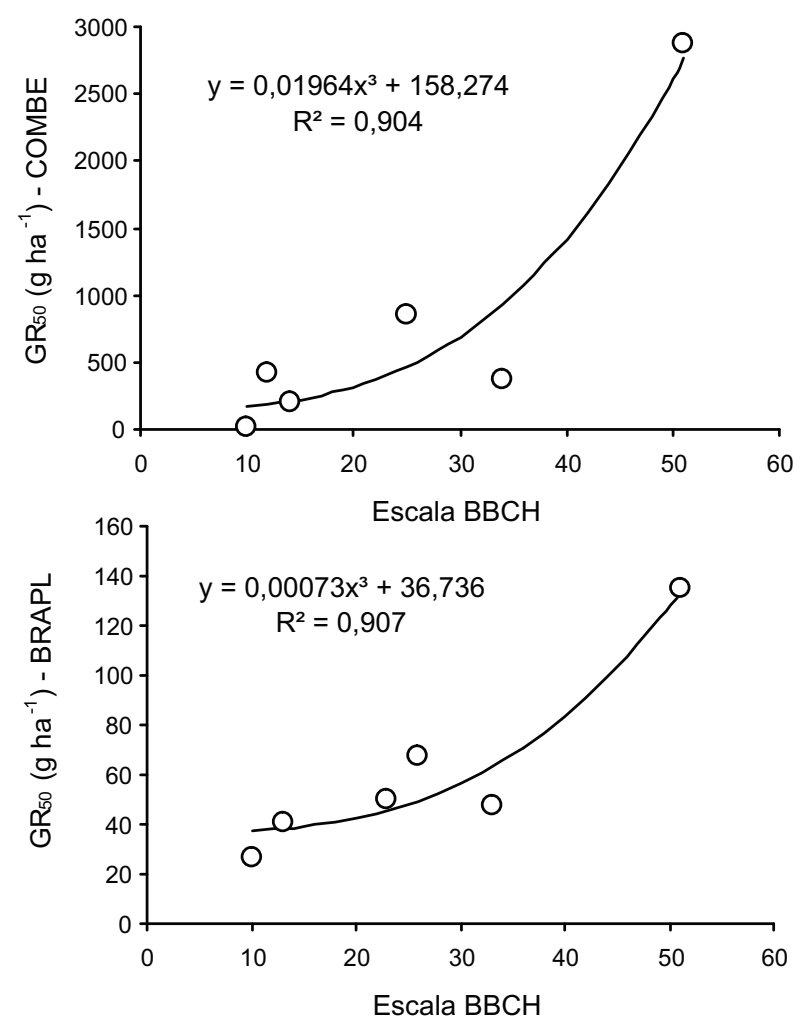

Figura 1 - Dose necessária para 50\% de redução na massa seca $\left(\mathrm{GR}_{50}\right)$ de Commelina benghalensis (COMBE) e Brachiaria plantaginea (BRAPL), ajustada ao desenvolvimento fenológico das plantas, avaliado segundo a escala $\mathrm{BBCH}$. Piracicaba SP, 2008 
glyphosate inferiores a $160 \mathrm{~g} \mathrm{ha}^{-1}$ (Figura 1). A elevada suscetibilidade do capim-marmelada ao glyphosate torna-se ainda mais evidente quando são considerados os valores de massa seca e área foliar no momento da aplicação (Tabela 1), notadamente superiores aos da trapoeraba. Ou seja, mesmo com maior acúmulo de massa e folhas, o capim-marmelada permanece altamente sensivel à ação tóxica do herbicida.

O fator de tolerância da trapoeraba ao glyphosate, calculado em função do capimmarmelada, teve variação entre 4 e 20, com equação $y=0,4112 x$ (Figura 2). Nota-se, portanto, que o desenvolvimento vegetativo da trapoeraba dificulta sobremaneira seu controle, de modo que a cada dez unidades de desenvolvimento BBCH têm-se plantas de trapoeraba quatro vezes mais tolerantes ao glyphosate. Nesse sentido, Christoffoleti et al. (2005) e Ribeiro (2008) também observaram interferência do estádio fenológico das plantas na eficácia do herbicida glyphosate, registrando menor controle para plantas mais desenvolvidas de azevém (Lolium multiflorum) resistente ao produto.

Conclui-se que o estádio de desenvolvimento da trapoeraba contribui significativamente para o grau de tolerância da espécie ao herbicida glyphosate, de modo que, comparativamente ao capim-marmelada, plantas de trapoeraba tornam-se quatro vezes mais tolerantes ao glyphosate a cada dez unidades de desenvolvimento fenológico na escala $\mathrm{BBCH}$.

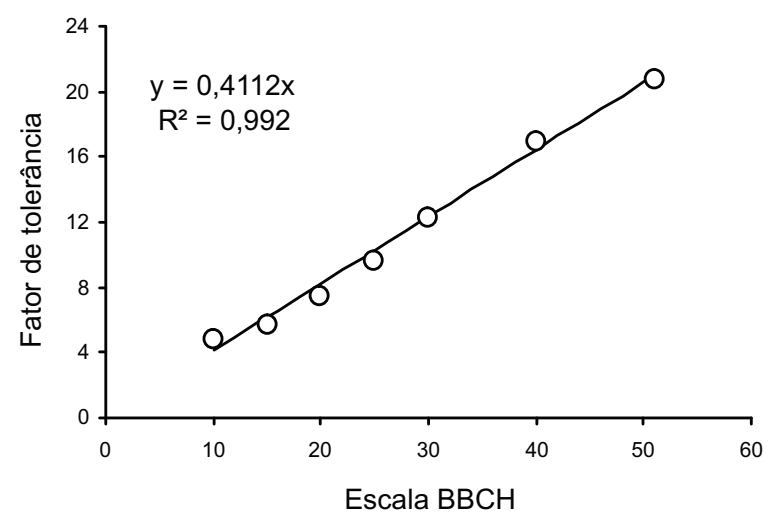

Figura 2 - Evolução do fator de tolerância de trapoeraba (Commelina benghalensis) em relação ao capim-marmelada (Brachiaria plantaginea), ajustada ao desenvolvimento fenológico das plantas, avaliado segundo a escala $\mathrm{BBCH}$. Piracicaba-SP, 2008.
Essas considerações possuem importante aplicabilidade prática, de modo a justificar medidas de controle químico em estádios iniciais do crescimento da trapoeraba, reduzindo a dose de glyphosate e, consequentemente, seu custo de aplicação, com diminuição na pressão de seleção sobre biótipos resistentes a esse herbicida e redução dos danos ao agroecossistema.

\section{AGRADECIMENTOS}

À Coordenação de Aperfeiçoamento de Pessoal de Nivel Superior (CAPES), pela bolsa de mestrado concedida ao primeiro autor.

\section{LITERATURA CITADA}

ASKEW, S. D.; SHAW, D. R.; STREET, J. E. Graminicide application timing influences red rice (Oryza sativa) control and seedhead reduction in soybean (Glycine max)

Weed Technol., v. 14, n. 1, p. 176-181, 2000.

BRIGHENTI, A. M.; OLIVEIRA, M. F. Biologia de plantas daninhas. In: OLIVEIRA JR, R. S.; CONSTANTIN, J.; INOUE, M. H. (Eds.). Biologia e manejo de plantas daninhas. Curitiba: Omnipax, 2011. p. 1-36.

CARVALHO, S. J. P. et al. Crescimento e desenvolvimento da planta daninha capim-camalote. Bragantia, v. 64, n. 4, p. 591-600, 2005a.

CARVALHO, S. J. P. et al. Curvas de dose-resposta para avaliação do controle de fluxos de emergência de plantas daninhas pelo herbicida imazapic. Planta Daninha, v. 23, n. 3 , p. $535-542,2005$ b

CHRISTOFFOLETI, P. J. Curvas de dose-resposta de biótipos resistente e suscetível de Bidens pilosa L. aos herbicidas inibidores da ALS. Sci. Agric., v. 59, n. 3, p. 513-519, 2002.

CHRISTOFFOLETI, P. J. et al. Alternative herbicides to manage Italian ryegrass (Lolium multiflorum Lam.) resistant to glyphosate at different phenological stages. J. Environ. Sci. Health, Part B, v. 40, n. 1, p. 59-67, 2005.

CHRISTOFFOLETI, P. J.; LÓPEZ-OVEJERO, R. F. Resistência de plantas daninhas a herbicidas: definições, bases e situação no Brasil e no mundo. In: CHRISTOFFOLETI, P. J. (Coord.). Aspectos de resistência de plantas daninhas a herbicidas. 3.ed. Piracicaba: Associação Brasileira de Ação a Resistência de Plantas aos Herbicidas, 2008. p. 9-29.

DIAS, A. C. R. et al. Competitiveness of alexandergrass or bengal dayflower with soybean. Planta Daninha, v. 28, n. 3, p. $515-522,2010$. 
DIAS, A. C. R. et al. Germinação de sementes aéreas pequenas de trapoeraba (Commelina benghalensis). Planta Daninha, v. 27, p. 931-939, 2009. (Número Especial)

FLECK, N. G. et al. Controle de papuã (Brachiaria plantaginea) em soja em função da dose e da época de aplicação do herbicida clethodim. Planta Daninha, v. 26, n. 2, p. 375-383, 2008.

FRIESEN, L. F. et al. Response of a chlorosulfuron resistant biotype of Kochia scoparia to sulfonylurea and alternative herbicides. Weed Sci., v. 41, n. 1, p. 100-106, 1993.

HALL, L. M.; STROMME, K. M.; HORSMAN, G. P. Resistance to acetolactate synthase inhibitors and quinclorac in a biotype of false cleavers (Galium spurium). Weed Sci., v. 46, n. 4 , p. $390-396,1998$.

HESS, M. et al. Use of the extended BBCH scale - general for the descriptions of the growth stages of mono- and dicotyledonous weed species. Weed Res., v. 37, n. 6, p. 433-441, 1997.

JOHNSON, G. A.; HOVERSTAD, T. R. Effect of row spacing and herbicide application timing on weed control and grain yield in corn (Zea mays). Weed Technol., v. 16, n. 3, p. 548-553, 2002.

KRUSE, N. D.; TREZZI, M. M.; VIDAL, R. A. Herbicidas inibidores da EPSPs: revisão de literatura. R. Bras. Herbic., v. 1, n. 2, p. 139-146, 2000.

LACERDA, A. L. S.; VICTORIA FILHO, R. Curvas doseresposta em espécies de plantas daninhas com o uso do herbicida glyphosate. Bragantia, v. 63, n. 1, p. 73-79, 2004.

LORENZI, H. Manual de identificação e controle de plantas daninhas: plantio direto e convencional. 5.ed. Nova Odessa: Instituto Plantarum, 2000. 381 p.

MACIEL, C. D. G. et al. Misturas em tanque com glyphosate para o controle de trapoeraba, erva-de-touro e capimcarrapicho em soja RR ${ }^{\circledR}$. R. Ceres, v. 58, n. 1, p. 35-42, 2011

MADSEN, K. H.; JENSEN, J. E. Weed control in glyphosate tolerant sugarbeet (Beta vulgaris L.). Weed Res., v. 35, n. 2, p. 105-111, 1995.
MONQUERO, P. A. et al. Absorção, translocação e metabolismo do glyphosate por plantas tolerantes e suscetíveis a este herbicida. Planta Daninha, v. 22, n. 3, p. 445-451, 2004.

PEREIRA, M. R. R. et al. Efeito de herbicidas sobre plantas de Brachiaria plantaginea submetidas a estresse hídrico. Planta Daninha, v. 28, p. 1047-1058, 2010. (Número Especial)

RIBEIRO, D. N. Caracterização da resistência ao herbicida glyphosate em biótipos da planta daninha Lolium multiflorum (Lam.). 2008. 102 f. Dissertação (Mestrado em Fitotecnia) - Escola Superior de Agricultura "Luiz de Queiroz", Universidade de São Paulo, Piracicaba, 2008.

ROCHA, D. C.; RODELLA, R. A.; MARTINS, D. Caracterização morfológica de espécies de trapoeraba (Commelina spp.) utilizando a análise multivariada.

Planta Daninha, v. 25, n. 4, p. 671-678, 2007.

RODRIGUES, B. N.; ALMEIDA, F. L. S. (Ed.). Guia de herbicidas. Londrina: Edição dos Autores, 2011. 697 p.

ROMAN, E. S. et al. Influência do orvalho e volume de calda de aplicação na eficácia do glyphosate na dessecação de Brachiaria plantaginea. Planta Daninha, v. 22, n. 3, p. 479-482, 2004.

SEEFELDT, S. S.; JENSEN, S. E.; FUERST, E. P. Log-logistic analysis of herbicide dose response relationship.

Weed Technol., v. 9, n. 1, p. 218-227, 1995.

STREIBIG, J. C.; RUDEMO, M.; JENSEN, J. E. Doseresponse curves and statistical models. In: STREIBIG, J. C.; KUDSK, P. (Ed.). Herbicide bioassay. Boca Raton: CRC Press, 1993. p. 30-35.

WEBSTER, T. M.; GREY, T. L. Growth and reproduction of bengal dayflower (Commelina benghalensis) in response to drought stress. Weed Sci., v. 56, n. 4, p. 561-566, 2008.

ZONETTI, P. C. et al. Growth and root lignification of susceptible and glyphosate-resistant soybean. Acta Sci. Agron., v. 33, n. 2, p. 291-295. 2011 